\title{
SIRT5 Directly Inhibits the PI3K/AKT Pathway in Prostate Cancer Cell Lines
}

\author{
SO YOUNG CHOI ${ }^{1 *}$, JU MI JEON ${ }^{1 *}$, ANN YAE NA ${ }^{1}, \mathrm{OH}_{\mathrm{KWANG}} \mathrm{KWON}^{1}$, \\ IN HYUK BANG ${ }^{2}$, YUN-SOK HA ${ }^{3}$, EUN JU BAE ${ }^{4}$, BYUNG-HYUN PARK ${ }^{2}$, \\ EUN HYE LEE ${ }^{5}$, TAE GYUN KWON ${ }^{3}$, JUN NYUNG LEE ${ }^{3 \#}$ and SANGKYU LEE ${ }^{1 \#}$ \\ ${ }^{1}$ BK21 Plus KNU Multi-Omics Based Creative Drug Research Team, College of Pharmacy, \\ Research Institute of Pharmaceutical Sciences, Kyungpook National University, Daegu, Republic of Korea; \\ ${ }^{2}$ Department of Biochemistry and Molecular Biology, \\ Chonbuk National University Medical School, Jeonbuk, Republic of Korea; \\ ${ }^{3}$ Department of Urology, School of Medicine, Kyungpook National University, Daegu, Republic of Korea; \\ ${ }^{4}$ College of Pharmacy, Chonbuk National University, Jeonbuk, Republic of Korea; \\ ${ }^{5}$ Joint Institute for Regenerative Medicine, Kyungpook National University, Daegu, Republic of Korea
}

\begin{abstract}
Background/Aim: Prostate cancer (PCa) is the most commonly diagnosed genital cancer in men globally. Among patients who develop advanced PCa, 80\% are affected by bone metastasis, with a sharp drop in survival rate. Despite efforts, the details of mechanisms of metastasis of $P C a$ remain unclear. SIRT5, an $N A D^{+}$-dependent deacylase, is hypothesized to be a crucial regulator of various cancers. The role of SIRT5 in cancer has not been extensively studied compared to other SIRTs. In this study, we showed significantly decreased levels of SIRT5 in PC-3M, a highly aggressive PC-3 cell variant. Materials and Methods: We characterized the differentially expressed proteins between parental and SIRT5 KO PC-3 cells using quantitative proteomics analysis. Results: A significant
\end{abstract}

This article is freely accessible online.

*These Authors contributed equally to this study.

\#These Authors jointly supervised this study.

Correspondence to: Sangkyu Lee, Ph.D., Associate Professor, College of Pharmacy, Research Institute of Pharmaceutical Sciences, Kyungpook National University, 80 Daehak-ro, Buk-gu 41566, Republic of Korea. Tel: +82 539508571, Fax: +82 539508557, e-mail: sangkyu@knu.ac.kr and Jun Nyung Lee, MD, Ph.D., Associate Professor, Department of Urology, School of Medicine, Kyungpook National University, 807 Hoguk-ro, Buk-gu, Daegu 41405, Republic of Korea. Tel: +82 532002675, Fax: +82 532002027, e-mail: ljnlover@gmail.com

Key Words: Prostate cancer, SIRT5, metastasis, PI3K/AKT pathway, IL-1B. increase in expression of interleukin-1 $\beta(I L-1 \beta)$ in SIRT5 KO cells was observed, and the PI3K/AKT/NF-kB signaling pathway was found significantly elevated in SIRT5 KO cells by the Gene Ontology annotation and KEGG pathway functional enrichment analysis. Moreover, we confirmed that SIRT5 can bind PI3K by immunoprecipitation analysis. Conclusion: This study is the first to demonstrate a relationship between SIRT5 and PCa metastasis, suggesting that SIRT5-mediated inhibition of the PI3K/AKT/NK-kB pathway is reduced for secondary metastasis from bone to other tissues.

Silent information regulator 2-like proteins (sirtuin, SIRT) are highly conserved proteins with nicotinamide adenine dinucleotide (NAD)-dependent deacylase activity and are classified as class III histone deacetylase enzymes $(1,2)$. There are seven types of SIRTs (1-7) in mammals, and they are known to be present in all organelles in cells and play a characteristic role (3). SIRTs have specific functions in cancer and normal cells. Numerous studies have revealed that SIRTs play critical roles in the progression of cancer and metastases by regulating pathways of angiogenesis, inflammation, and epithelial-to-mesenchymal transition (46). Among the seven SIRTs, SIRT5 is characterized as a $\mathrm{NAD}^{+}$-dependent lysine deacetylase, demalonylase, desuccinylase, and deglutarylase (7). SIRT5 is also involved in cell metabolism, including glycolysis, tricarboxylic acid cycle, fatty acid oxidation, nitrogen metabolism, pentose phosphate pathway, antioxidant defense, and apoptosis (8). Particularly, SIRT5 plays a role in tumorigenesis by regulating desuccinylation involved in specific enzymatic activities $(9,10)$. SIRT5 also has important regulatory roles in tumor progression in the liver and gastric cancer $(11,12)$. 
Prostate cancer ( $\mathrm{PCa}$ ) is the most commonly diagnosed cancer type in men and is the third-leading cause of deaths related to cancer (13). The number of patients with PCa is expected to increase due to population aging and expansion. Numerous reports have shown that cancer metastasis is the primary cause of malignant characteristics, leading to a diverse change of metastatic regulatory factors (14). PCa can spread to various tissues, especially the lymph node and bone (15). According to the results of a previous study, $\mathrm{PCa}$ first metastasizes to the bone and then to other organs (16), which means that the progression from the bone to other metastatic sites is associated with decreased survival in late stages of PCa.

In this study we used the PC- 3 cell line, where prostate cancer cells have metastasized to the bone, a cell line most commonly used in prostate cancer research (17). PC-3M cell line is a variant cell line of PC-3 cells having highly metastatic potential compared to PC-3 cells (18). Because PC-3M exhibits higher migration and invasion than PC-3, it can represent prostate cancer cells in the second metastasis from the bone, which is the primary metastatic organ of prostate cancer, to other organs. In our previous studies, it was observed that SIRT5 was significantly inhibited in PC3M compared to PC-3. To study the regulation mechanism of SIRT5 related to metastasis, a SIRT5 KO cell line was constructed, and proteins regulated by SIRT5 were identified using comparative quantitative proteomics. Consequently, it was observed that the PI3K/AKT pathway is regulated using SIRT5, which is related to inhibiting tumor cell growth, migration, and invasion and modulating the innate and adaptive immune responses.

\section{Materials and Methods}

Cell culture and stable isotope labeling using amino acids in cell culture (SILAC) labeling. The human prostate cancer cell lines PC-3, PC-3M, and PC-3 SIRT5 KO were obtained from the Korean Cell Line Bank (Seoul, Republic of Korea). Cell lines were cultured under standard conditions according to the manufacturer. Briefly, PCa cell lines were cultured in RPMI 1640 medium (Pierce HyClone, Fremont, CA, USA) containing $10 \%$ fetal bovine serum (GE Healthcare HyClone, Logan, UT, USA) and $1 \%$ penicillin and streptomycin (Gibco, Rockville, MD, USA). The cells were incubated at $37^{\circ} \mathrm{C}$ with $5 \% \mathrm{CO}_{2}$ in a humidified incubator. PC-3 cells were cultured and labeled using the SILAC kits with $\mathrm{Lys}^{4}\left(\mathrm{D}_{4}\right)$ and $\mathrm{Arg}^{6}$ $\left({ }^{13} \mathrm{C}_{6}\right)$ ("Medium") labeling for LC-MS/MS analysis. After confirming that the SILAC labeling efficiency was $>98 \%$, the cells were lysed with RIPA buffer, and lysates were quantified using BCA assay. Next, we mixed the two cell lines equally (300- $\mu$ g each) and performed reduction and alkylation using 15-mM DTT and 60-mM IAA. Proteins were digested into peptides using trypsin overnight at $37^{\circ} \mathrm{C}$. Then, high-pH reverse phase (HP-RP) fractionation and off-gel fractionation was performed according to the manufacturer's protocol.

Construction of SIRT5 knockout cell lines using the CRISPR-Cas9 gene-editing system. We used the clustered regularly interspaced short palindromic repeats (CRISPR) and CRISPR-associated protein-9 endonuclease (Cas9) system to knockout SIRT5 from the cell lines. According to the manufacturer's instructions, PC-3 cells were transfected with either the control or Sirt5 CRISPR-Cas9 plasmids (Sigma-Aldrich, St. Louis, MO, USA). The transfection efficiency was determined using the green fluorescent protein (GFP) signal. After $24 \mathrm{~h}, \mathrm{PC}-3$ cells expressing GFP were sorted using FACS Aria III (BD, Franklin Lakes, NJ, USA) and were maintained with complete media. Deletion of Sirt 5 by the CRISPR-Cas9 Sirt5 plasmid but not the control plasmid was confirmed using Western blot analysis.

Western blotting. PCa cell lines were lysed using RIPA buffer (Thermo, Waltham, MA, USA), and protein concentrations were measured using the BCA assay (Thermo). Briefly, samples were separated by $10 \%$ SDS-PAGE for $90 \mathrm{~min}$ and transferred to PVDFblotting membranes $(0.2-\mu \mathrm{m}$ pore size, GE Healthcare, Chalfont St. Giles, UK) for $2 \mathrm{~h}$. The membrane was blocked in 5\% BSA for 2 $\mathrm{h}$, and primary antibodies were used to collect the target proteins overnight at $4^{\circ} \mathrm{C}$. After incubation with HRP-conjugated secondary antibody for $1 \mathrm{~h}$, the membranes were washed thrice in TBS-T (Tris-buffered saline and $0.1 \%$ Tween 20) for $15 \mathrm{~min}$. ECL Prime Western blotting detection reagent (GE Healthcare) was used to visualize blots. The primary antibodies were followed as SIRT5 (Abcam, Cambridge, UK) and GAPDH (Thermo).

Protein immunoprecipitation. PI3K (Abcam) or mouse $\mathrm{IgG}$ (SantaCruz Biochemicals, Dallas, TX, USA) antibody and protein $\mathrm{G}$ magnetic nanobeads (bioneer, Daejeon, Republic of Korea) were mixed and incubated in a rotator for $30 \mathrm{~min}$ at room temperature and then washed with PBS. Then, $500 \mathrm{ng}$ to $1 \mathrm{mg}$ of protein were mixed with nanobeads and then incubated in a rotator for $1 \mathrm{~h}$ at room temperature and then washed with PBS. The elution buffer was added and eluted in a vortex for $10 \mathrm{~min}$ and then nanobeads were removed. Finally, the samples were incubated for $10 \mathrm{~min}$ in $4 \%$ SDS at $95^{\circ} \mathrm{C}$.

Real-time quantitative polymerase chain reaction ( $R T-q P C R)$. Total RNA was isolated from PC-3 and SIRT5-KO cells using an RNA Cell Miniprep kit (Promega, Fitchburg, WI, USA) according to the manufacturer's instructions. cDNA synthesis was performed using a GoScript Reverse Transcription kit (Promega) and then used as a qPCR template with FASTStart Essential DNA Green Master mix (Roche, Basel, Switzerland). The primer sequences were as follows: IL-1b forward 5'-CTTCGAGGCACAAGGCACAA-3' and reverse 5'-TTCACTGGCGAGCTCAGGTA-3' and ACTB forward 5'-TGCG CCGTTCCGAAAGTT-3' and reverse 5'-GCGCCGCTGGG TTTTATAG-3'.

LC-MS/MS analysis. Samples were analyzed via high-resolution accurate mass spectrometry using LTQ-Velos orbitrap (Thermo Fisher Scientific) connected with an Eksigent nano-LC (SCIEX, Framingham, MA, USA). Then, 5- $\mu$ g peptides was dissolved in $10-\mu \mathrm{l}$ solution A ( $98 \%$ water in $0.1 \%$ formic acid), and $2 \mu$ sample was automatically loaded onto a homemade $\mathrm{C} 12$ reversed-phase analytical column $(75-\mu \mathrm{m}$ X $120 \mathrm{~mm}$, Jupiter C12 resin, $90 \AA$ pore size, $4-\mu \mathrm{m}$ particle size; Phenomenex, Torrance, CA, USA) by the autosampler nano-LC system. The 60 min linear gradient was $3 \%$ solvent B $(100 \%$ $\mathrm{ACN}$ in $0.1 \%$ formic acid) for $2 \mathrm{~min}$ and $3 \%-23 \%$ solvent $\mathrm{B}$ for 50 min, $23 \%-90 \%$ solvent $\mathrm{B}$ for five minutes, and $90 \%$ for $5 \mathrm{~min}$ at a constant flow rate of $300 \mathrm{nl} / \mathrm{min}$. LTQ-Velos orbitrap with nanoelectrospray ionization (nano-ESI) was conducted using the 

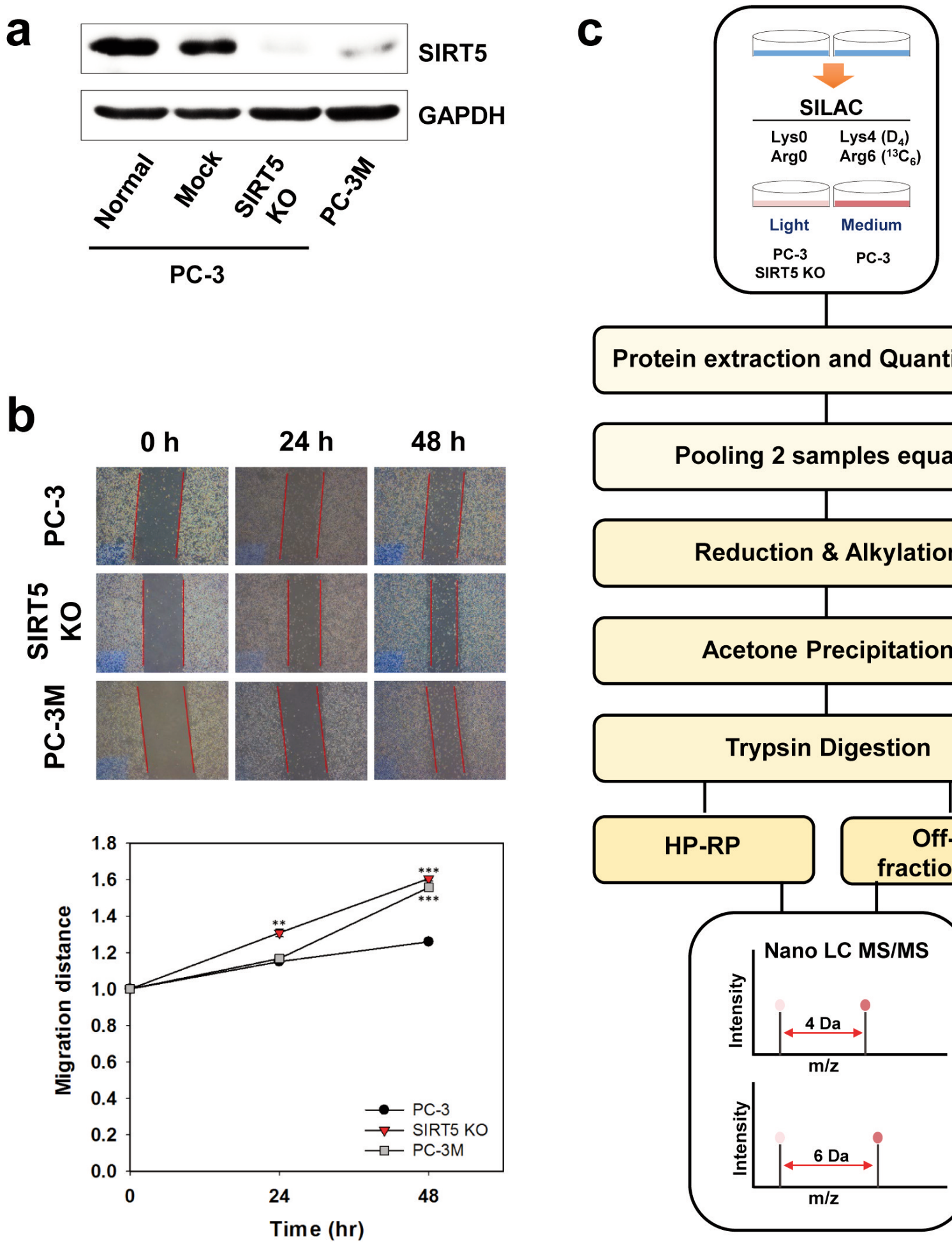

\section{Protein extraction and Quantification}

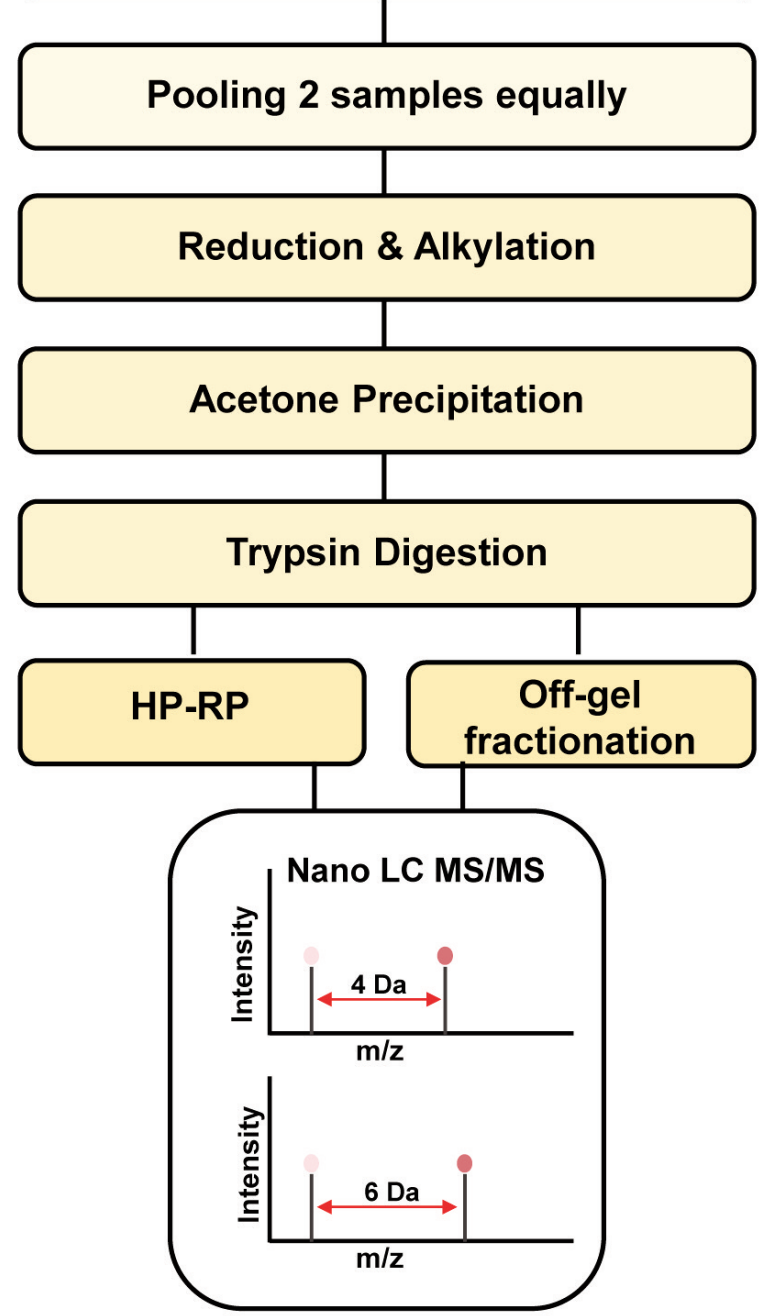

Figure 1. Workflow for quantitative proteomics to identify differentionally expressed proteins related to SIRT5. (a) Western blot of SIRT5 in prostate cancer cells (Normal: nontreated PC-3 cells, Cont: Cas9vehicle-treated PC-3 cells, SIRT5 KO: PC-3 cells knocked out for SIRT5). (b) Migration assay of normal, SIRT5 KO, PC-3M cells (24 $h$ and $48 h, n=3),(* * p<0.01, * * * p<0.001)$. (c) Schematic figure of the workflow for the relative quantification of proteins extracted from prostate cancer cells. 
a
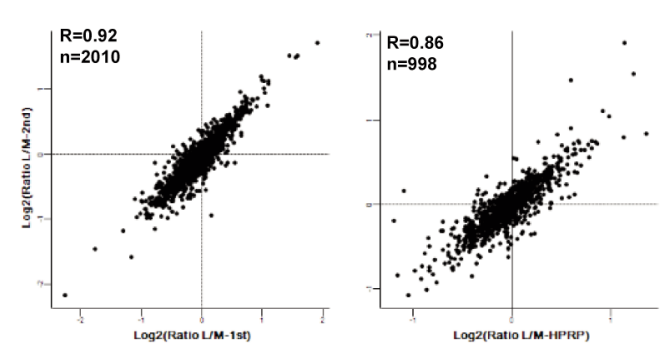

b

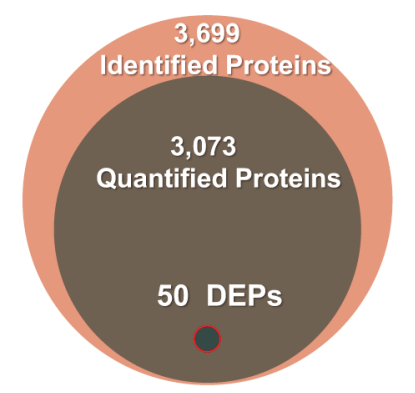

C

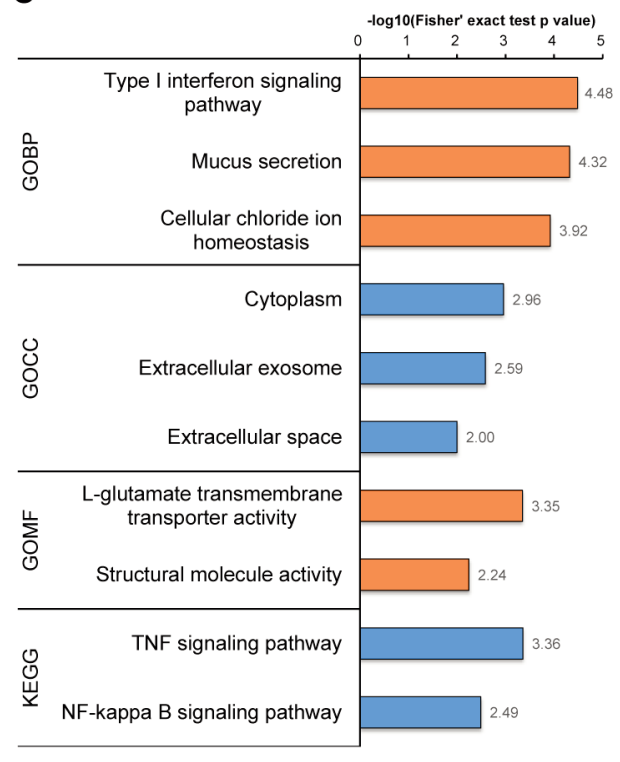

d

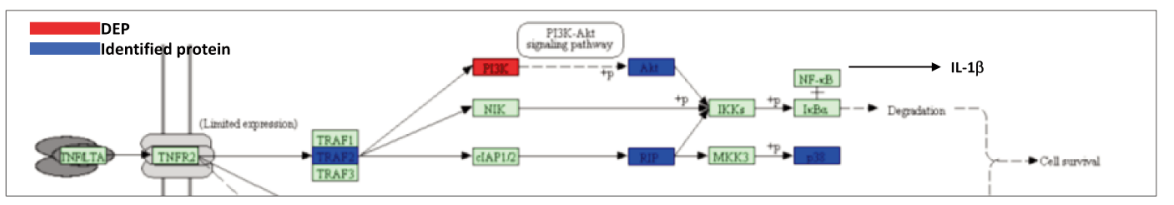

Figure 2. Characterization of differentially expressed protein in PC-3 SIRT5 KO cells. (a) Pearson correlation coefficient (PCC) score between 1st batch and 2nd batch, HP-RP and Off-gel fractionation (b) Venn diagram of the identified proteins, quantified proteins and DEPs. (c) Functional enrichment analysis of proteins up-regulated in PC-3 SIRT5 KO cells based on GO annotation and KEGG pathway analysis using DAVID. (d) PI3K/AKT/NF-kB pathway in KEGG mapper.

following settings: nanospray source voltage $1.8 \mathrm{kV}$; full MS range $300-1,800 \mathrm{~m} / \mathrm{z}$ with 70,000 resolution (at $200 \mathrm{~m} / \mathrm{z}$ ); top 20 datadependent mode with Collision-Induced Dissociation; normalized collision energy at $28 \%$; and $1.7 \mathrm{~m} / \mathrm{z}$ isolation width. To improve the full MS accuracy, the lock-mass ion from ambient air $(\mathrm{m} / \mathrm{z}$ 445.120024) was applied. Mass spectrometry proteomics data have been deposited on the ProteomeXchange Consortium via the PRIDE (19) partner repository with the dataset identifier PXD012735.

Protein identification and quantification. The MS spectra were searched using the MaxQuant 1.5.1.0 protein search engine, which can identify and quantify proteins (20). For SILAC-based quantification, Lys0 $\left({ }^{12} \mathrm{C}_{6}{ }^{14} \mathrm{~N}_{2}\right)$ and $\operatorname{Arg} 0\left({ }^{12} \mathrm{C}_{6}{ }^{14} \mathrm{~N}_{4}\right)$ were defined as light labels (L), and Lys4 $\left(\mathrm{D}_{4}\right)$ and $\operatorname{Arg} 6\left({ }^{13} \mathrm{C}_{6}\right)$ were specified as medium $(\mathrm{M})$. The UniprotKB Human database (containing 71,772 protein sequences) was used with trypsin/p as the digestive enzyme. Cysteine carbamidomethylation was set as a fixed modification, and methionine oxidation and protein $N$-terminal acetylation were considered variable modifications. The other parameters were set to default values. Potential contaminants and proteins only identified by a modification site were removed from the search results. Furthermore, the false discovery rate was set below $1 \%$ to improve protein identification. The SILAC ratio was calculated using L/M (PC-3 SIRT5 KO cell line/PC3 cell line) with a minimum ratio count of two and then transformed to $\log 2$-fold changes. The other parameters in MaxQuant were set to default values. The ratios obtained were normalized to the median, and a $\log 2$-transformation of the protein ratios was obtained.

Bioinformatics analysis. Scatter plots, histograms, and volcano plots were constructed using Perseus, according to the manufacture's procedure (21). Thus, enrichment analyses with Gene Ontology (GO) classification, KEGG pathway, and InterPro for protein domains were conducted by DAVID (22). The web-based STRING was used for protein-protein interactions with a score up to 0.9 (23).

Data and statistics. All experiments were repeated at least thrice, and data are expressed as mean values \pm standard errors. One-way ANOVA was used to compare statistical significance of data. To determine the significant differences, statistical analysis was conducted using IBM SPSS Statistics version 21. $p$-Values $<0.05$ were considered statistically significant and are indicated with asterisks. $(* * p<0.01, * * * p<0.001)$. 
Table I. The list of down-regulated proteins in SIRT5 KO compared to PC-3 cells.

\begin{tabular}{|c|c|c|c|c|c|}
\hline $\begin{array}{l}\text { Protein } \\
\text { accession }\end{array}$ & Protein description & $\begin{array}{c}\text { Log2 ratio* } \\
\text { (SIRT5 KO } \\
\text { ss. PC-3) }\end{array}$ & $\begin{array}{c}\text { Number of } \\
\text { peptides }\end{array}$ & $\begin{array}{c}\text { Type } \\
\text { (SIRT5 KO } \\
\text { vs. PC-3) }\end{array}$ & Gene name \\
\hline P52943 & Cysteine-rich protein 2 & -3.077 & 4 & Down & CRIP2 \\
\hline Q15847 & Adipogenesis regulatory factor & -2.231 & 2 & Down & $A D I R F$ \\
\hline Q15848 & Thromboxane-A synthase & -2.003 & 2 & Down & $T B X A S 1$ \\
\hline Q15849 & Neurofilament light polypeptide & -1.645 & 3 & Down & NEFL \\
\hline Q15850 & Nestin & -1.608 & 35 & Down & NES \\
\hline Q15851 & PI-PLC X domain-containing protein 3 & -1.544 & 2 & Down & $P L C X D 3$ \\
\hline Q15852 & Interferon-stimulated gene $20 \mathrm{kDa}$ protein & -1.493 & 3 & Down & ISG20 \\
\hline Q15853 & Leukocyte elastase inhibitor & -1.447 & 8 & Down & SERPINB1 \\
\hline Q15854 & Keratin, type I cytoskeletal 18 & -1.322 & 16 & Down & KRT18 \\
\hline Q15855 & Centrosome-associated protein CEP250 & -1.191 & 2 & Down & CEP250 \\
\hline Q15856 & Striatin & -1.165 & 3 & Down & STRN \\
\hline Q15857 & Anterior gradient protein 2 homolog & -1.162 & 3 & Down & $A G R 2$ \\
\hline Q15858 & Calcium-binding mitochondrial carrier protein Aralar2 & -1.138 & 5 & Down & SLC25A13 \\
\hline Q15859 & Bifunctional epoxide hydrolase 2 & -1.137 & 1 & Down & EPHX2 \\
\hline Q15860 & Tubulin beta- 3 chain & -1.130 & 3 & Down & TUBB3 \\
\hline Q15861 & Deoxynucleoside triphosphate triphosphohydrolase SAMHD1 & -1.120 & 4 & Down & SAMHD1 \\
\hline Q15862 & Actin-binding LIM protein 3 & -1.106 & 2 & Down & ABLIM3 \\
\hline Q15863 & Intercellular adhesion molecule 1 & -1.080 & 2 & Down & ICAM1 \\
\hline Q15864 & Zinc finger protein 185 & -1.066 & 11 & Down & ZNF185 \\
\hline Q15865 & Creatine kinase B-type & -1.060 & 3 & Down & $C K B$ \\
\hline Q15866 & Solute carrier organic anion transporter family member $4 \mathrm{~A} 1$ & -1.038 & 1 & Down & SLCO4A1 \\
\hline Q15867 & Septin-8 & -1.025 & 2 & Down & SEPT8 \\
\hline Q15868 & Vesicle-associated membrane protein 8 & -1.016 & 3 & Down & VAMPS \\
\hline Q15869 & Claudin-4 & -1.015 & 1 & Down & CLDN4 \\
\hline Q15870 & 2-5-oligoadenylate synthase 3 & -1.012 & 2 & Down & OAS3 \\
\hline Q15871 & Interferon-induced $35 \mathrm{kDa}$ protein & -1.000 & 1 & Down & IFI35 \\
\hline
\end{tabular}

*The ratios obtained were normalized to the median and a $\log 2$-transformation of the protein ratios was obtained.

\section{Results}

Down-regulation of SIRT5 in advanced human prostate cancer. Previously, we identified that SIRT5 selectively decreased in PC-3M compared with PC-3 cells (Figure 1a). The PC-3M cell line is a subline of PC-3 that was removed from a PC3-induced mouse tumor (24). Because PC-3M exhibits enhanced metastasis compared with PC-3, it can be deduced that reduced SIRT5 expression in PC-3M may be associated with metastasis. Next, we used a migration assay with PC-3, SIRT5 KO, and PC-3M cells to assess whether the migratory ability is dependent on SIRT5. Interestingly, SIRT5 KO cells migrated significantly faster than PC-3 cells as measured by TScratch software, suggesting that SIRT5 regulates the migration of PCa cells (Figure 1b).

Identification of SILAC-labeled prostate cancer cell proteins by nano-LC-MS/MS. To identify protein markers of increased metastasis upon SIRT5 reduction in prostate cancer cells, we used an MS-based SILAC approach for quantitative analysis of the PC-3 proteome compared with that of SIRT5 KO cells
(Figure 1c). PC-3 and PC-3 SIRT5 KO cells were grown in SILAC media and normal RPMI media, respectively. In this experiment, we identified 3,699 proteins and quantified 3,073 proteins using the MaxQuant database search program. The technical duplicates were verified using a scatter plot for the correlation analysis, showing a Pearson correlation coefficient (PCC) of 0.92 between duplicated experiments (Figure 2a). Among these, 50 proteins of SIRT5 KO were identified as differentially expressed proteins (DEPs) compared to PC-3 (Figure 2b). Ratios were normalized according to the median.

The DEPs were categorized by relative protein quantification analysis (Figure 2b). DEPs showing greater than two-fold reduction (26 proteins) in SIRT5 KO cells were down-regulated compared to PC-3 (Table I), whereas DEPs with greater than a two-fold increase (24 proteins) were considered up-regulated proteins (Table II). From the DEP protein list, we discovered known factors related to metastasis regulation in various cancers. Cysteine-rich protein 2 (CRIP2), which regulates angiogenesis and invasion in cancer by suppressing NF-kB-mediated promotion of angiogenesis, was the most down-regulated protein in SIRT5 $\mathrm{KO}$ cells at a ratio 
Table II. The list of up-regulated proteins in SIRT5 KO compared to PC-3 cells.

\begin{tabular}{|c|c|c|c|c|c|}
\hline $\begin{array}{l}\text { Protein } \\
\text { accession }\end{array}$ & Protein description & $\begin{array}{c}\text { Log2 ratio* } \\
\text { (SIRT5 KO } \\
\text { vs. PC-3) }\end{array}$ & $\begin{array}{c}\text { Number of } \\
\text { peptides }\end{array}$ & $\begin{array}{c}\text { Type } \\
\text { (SIRT5 KO } \\
\text { vs. PC-3) }\end{array}$ & Gene name \\
\hline Q15872 & Sodium-dependent phosphate transporter 1 & 1.003 & 2 & Up & SLC20A1 \\
\hline Q15873 & Metallothionein-2 & 1.006 & 3 & Up & $M T 2 A$ \\
\hline Q15874 & Isochorismatase domain-containing protein 2 , mitochondrial & 1.006 & 3 & Up & ISOC2 \\
\hline Q15875 & RNA pseudouridylate synthase domain-containing protein 3 & 1.010 & 1 & $\mathrm{Up}$ & RPUSD3 \\
\hline Q15876 & Activator of basal transcription 1 & 1.016 & 2 & Up & $A B T 1$ \\
\hline Q15877 & Sulfiredoxin-1 & 1.031 & 2 & Up & SRXN1 \\
\hline Q15878 & Glutathione peroxidase & 1.031 & 2 & Up & $G P X 4$ \\
\hline Q15879 & Neuronal calcium sensor 1 & 1.037 & 1 & $\mathrm{Up}$ & NCS1 \\
\hline Q15880 & Peptide deformylase, mitochondrial & 1.040 & 1 & Up & $P D F$ \\
\hline Q15881 & Centrosomal protein of $85 \mathrm{kDa}$ & 1.058 & 1 & Up & CEP85 \\
\hline Q15882 & Protein HEXIM1 & 1.095 & 2 & Up & HEXIM1 \\
\hline Q15883 & Serpin B4 & 1.128 & 1 & $\mathrm{Up}$ & SERPINB4 \\
\hline Q15884 & AP-1 complex subunit sigma-2 & 1.136 & 1 & Up & $A P 1 S 2$ \\
\hline Q15885 & Ubiquitin carboxyl-terminal hydrolase & 1.137 & 1 & Up & USP11 \\
\hline Q15886 & Phosphatidylinositol 3-kinase regulatory subunit beta & 1.176 & 1 & Up & $P I K 3 R 2$ \\
\hline Q15887 & Proteasome activator complex subunit 4 & 1.268 & 3 & $\mathrm{Up}$ & PSME4 \\
\hline Q15888 & E3 ubiquitin-protein ligase NEDD4-like & 1.269 & 8 & Up & $N E D D 4 L$ \\
\hline Q15889 & Collagen alpha-2(VI) chain & 1.401 & 8 & Up & COL6A2 \\
\hline Q15890 & Discoidin, CUB and LCCL domain-containing protein 2 & 1.518 & 3 & Up & $D C B L D 2$ \\
\hline Q15891 & Piezo-type mechanosensitive ion channel component 1 & 1.523 & 2 & $\mathrm{Up}$ & PIEZO1 \\
\hline Q15892 & Urokinase-type plasminogen activator & 1.647 & 6 & Up & $P L A U$ \\
\hline Q15893 & CCAAT/enhancer-binding protein beta & 1.860 & 3 & Up & $C E B P B$ \\
\hline Q15894 & Excitatory amino acid transporter 1 & 2.032 & 3 & $\mathrm{Up}$ & $S L C 1 A 3$ \\
\hline Q15895 & Interleukin- 1 beta & 2.154 & 4 & Up & $I L 1 B$ \\
\hline
\end{tabular}

*The ratios obtained were normalized to the median and a $\log 2$-transformation of the protein ratios was obtained.

of -3.077 (25). Neurofilament light polypeptide (NEFL) is a known tumor suppressor in the head and neck squamous carcinoma via apoptosis induction and was down-regulated at a ratio of -1.645 in SIRT5 KO (26).

Numerous previous studies have analyzed proteins upregulated in SIRT5 KO cells. CCAAT/enhancer-binding protein beta (CEBPB, ratio +1.860 in SIRT5 KO) is a CEBP family member that acts as a transcription factor controlling normal tissue proliferation and differentiation (27). Table II shows that interleukin-1 beta (IL-1 $\beta$ ) was highly up-regulated in SIRT5 KO cells compared with PC-3 cells. IL- $1 \beta$ is a proinflammatory cytokine identified as an initiator of bone metastasis in primary breast cancer cells (28). These results are essential evidence that IL- $1 \beta$ may be involved in the aggressiveness of $\mathrm{PCa}$. Moreover, several proteasome proteins related to protein homeostasis were increased in SIRT5 KO cells, including E3 ubiquitin-protein ligase NEDD4-like (Nedd4-like E3) (ratio: +1.269 ), proteasome activator complex subunit 4 (ratio: +1.268 ), and ubiquitin carboxyl-terminal hydrolase (UHC) (ratio: +1.137). Given that dysregulation of the proteasome is linked to tumorigenesis, this result also needs to be further investigated in relation to the aggressiveness of prostate cancer.
To further investigate the mechanisms related to cell migration and invasion in SIRT5 KO cells, we performed functional enrichment analysis with Gene Ontology (GO), KEGG pathway, and InterPro by calculating Fisher's exact test $p$-values (Figure 2c). Using DAVID, we found DEPs related to the type 1 interferon signaling pathway in the immune system. Moreover, Gene Ontology Cellular Component analysis showed that the most enriched proteins are localized in the cytoplasm. Interestingly, KEGG pathway results identified the TNF- $\alpha$ signaling pathway and NF-kB signaling pathway as predicted pathways for the DEPs of SIRT5 KO cells compared with PC-3.

KEGG mapper showed that the pathway most related to our DEPs was the PI3K/AKT/NF-kB signaling pathway (Figure 2d). Therefore, we fixated on the PI3K/AKT/NF-kB signaling pathway for its role in tumor growth, migration, and invasion in various cancers. We identified four DEPs (intercellular adhesion molecule 1, ratio: -1.080 ; phosphatidylinositol 3kinase regulatory subunit beta, ratio: 1.176; CCAAT/enhancerbinding protein beta, ratio: 1.86 ; and interleukin-1 beta, ratio: 2.154) in the TNF-a signaling pathway and two DEPs (urokinase-type plasminogen activator [ratio: 1.647] and IL$1 \beta$ ) in the NF-kB signaling pathway. 
a

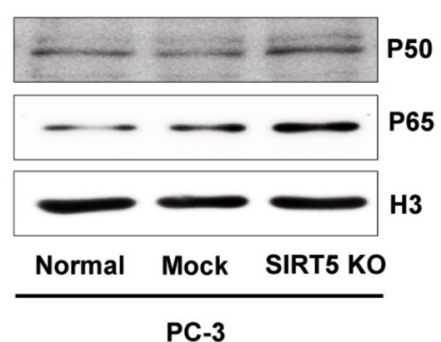

b

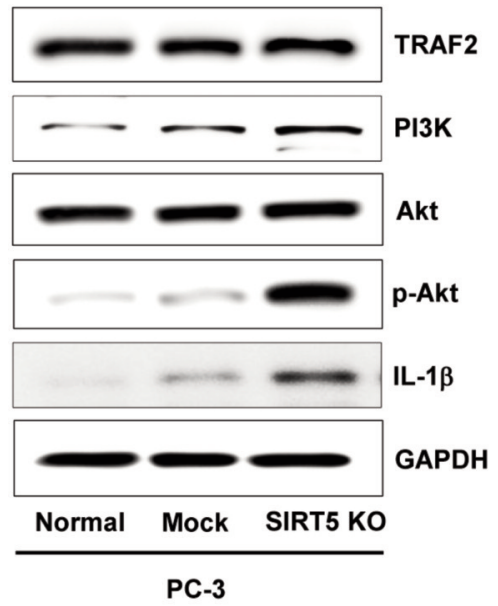

f

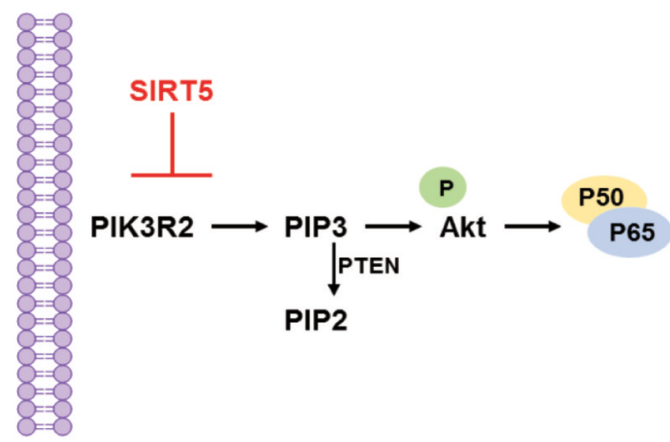

C

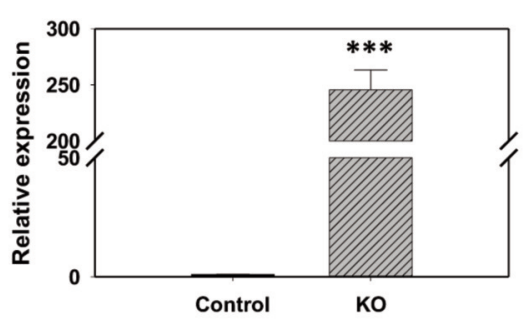

d

IP

IP

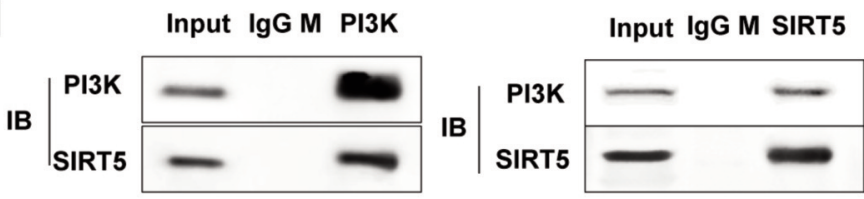

e

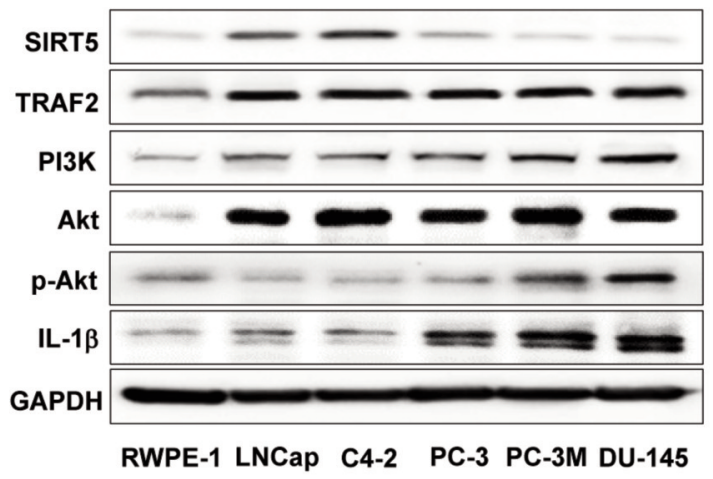

Prostate cancer cell-lines

Figure 3. Confirmation of the regulatory role of SIRT5 in the PI3K/AKT/NF-kB signaling pathway. (a) Western blots of nuclear proteins related to the PI3K/AKT/NF-kB signaling pathway. Histone H3 was used as a loading control of nuclear proteins. (b) Western blots of cytoplasmic proteins related to the PI3K/AKT/NF- $k B$ signaling pathway. (c) The level of IL-1 $\beta$ mRNA according to RT- $q$ PCR in PC-3 and SIRT5 KO cells ( $n=3$ ), $(* * * p<0.001)$. (d) The result of IP using a PI3K antibody on PC-3 and SIRT5 KO. (e) Western blot of the PI3K/AKT/NF-kB signaling pathway in prostate cancer cell lines. (f) Putate mechanism of the relation between SIRT5 and the PI3K/AKT/NF-kB signaling pathway.

SIRT5 regulates metastasis of prostate cancer by inhibiting the $P I 3 K / A K T / N F-k B$ signaling pathway. Furthermore, we extracted nuclear proteins from PC-3 and SIRT5 KO cells to assess levels of p50 and p65 as members of the NF-kB signaling pathway; both proteins were discovered to be increased in SIRT5 KO cells (Figure 3a). Based on these results, we speculated that SIRT5 can affect the PI3K/AKT/NF-kB signal to regulate $\mathrm{PCa}$ metastasis. To confirm the results, we used western blot of whole-cell lysates to rate TNF receptor-associated factor 2 (TRAF2, ratio: -0.083), PI3K, AKT (ratio: 0.130), p-AKT, and IL-1 $\beta$ (ratio: +2.154) expression, along with GAPDH (ratio: 0.016 ) as the loading control (Figure $3 \mathrm{~b}$ ). As expected, PI3K, p-AKT, and IL-1 $\beta$ levels, but not TRAF2, were increased in SIRT5 KO cells. 
Previous studies have shown that $\mathrm{NF}-\mathrm{kB}$ regulates transcription of IL- $1 \beta$, which is a downstream protein that regulates the aggressiveness of prostate cancer and other cancers (29). To examine whether the mRNA level of IL-1 $\beta$ was increased in SIRT5 KO cells, we conducted RT-qPCR and confirmed increased levels of IL-1 $\beta$ in SIRT5 KO cells, suggesting that elevated NF-kB activity positively regulated the transcriptional level of IL-1 $\beta$ (Figure $3 \mathrm{c}$ ). This result is consistent with our quantitative proteomics analysis.

According to KEGG mapper, TRAF2 of the NF-kB signaling pathway was not altered, but PI3K was significantly increased in SIRT5 KO cells. Based on these results, it is assumed that SIRT5 regulates the pathway by interacting with PI3K. When we investigated the interaction between PI3K and SIRT5 using immunoprecipitation (IP) with a PI3K IgG antibody, SIRT5 was found to interact with PI3K directly (Figure 3d). The possibility that SIRT5 is associated with the progression and metastasis of prostate cancer through the regulation of $\mathrm{PI} 3 \mathrm{~K} / \mathrm{AKT} / \mathrm{NF}-\mathrm{kB}$ signaling was evaluated in various prostate cancer cell lines (Figure $3 \mathrm{e}$ ).

The SIRT5 and AKT pathways were evaluated in the immortalized human normal prostate cells, RWPE-1 cells (30), and five PCa cell lines and are as follows; 1) androgen-sensitive LNCaP cells and insensitive C4-2 cells (31), as LNCaP's metastatic in vivo-derived variant; 2) bone metastatic PCa cells, including PC-3 and PC-3M as highly metastatic subline derived from PC-3 cells; and 3) brain metastatic PCa cells as DU-145 cells. SIRT5 decreased from C4-2 cells to PC-3 cells, that is, before and after metastasis, and PI3K increased slightly as the progression from LNCap to DU-145 cells. Moreover, as PI3K increased, AKT remained unchanged, whereas p-AKT increased in PC-3M and DU-145. This showed the same trend as in PC3 SIRT5 KO cells in Figure 3b, indicating that SIRT5 and pAKT had an inverse correlation. With these data, we speculated that SIRT5 can regulate the PI3K/AKT/NF-kB signaling pathway by directly blocking PI3K to induce overexpression of IL-1 $\beta$, which increases cell migration and invasion. The schematic representation of a putative mechanism of the SIRT5/PI3K signaling pathway for regulating cell migration and invasion in PC-3 cells is shown in Figure $3 \mathrm{f}$.

\section{Discussion}

SIRTs are class III histone deacetylases that are involved in diverse cellular processes inside and outside the mitochondria of normal and diseased cells $(1,2)$. Particularly, SIRTs have important roles in cancer initiation, progression, and metastases by regulating differentiation, cell adhesion, cell to cell communication, and inflammation (32). Among the SIRTs, the role of SIRT5 in cancer has not been extensively studied. It was recently discovered that SIRT5 may be involved in metabolic reprogramming to support the anabolic demands of rapidly dividing neoplastic cells (8). Sirt5 mRNA expression is detectable across various wide cancers, suggesting its function as an oncogene. For example, Sirt5 mRNA and protein expression are elevated in human lung cancer and are associated with poor survival in nonsmall cell lung cancer (NSCLC) (33). Moreover, Sirt5 mRNA expression was markedly increased in invasive breast tumors and B cell malignancy (34). In contrast, Sirt5 mRNA expression was significantly decreased in endometrial cancer and head and neck squamous cell carcinoma (35).

Based on quantitative comparative proteomics analysis between PC-3 and PC-3 SIRT5 KO cells, we identified DEPs in SIRT5 $\mathrm{KO}$ cells related to the PI3K/AKT/NF-kB signaling pathway using KEGG mapper software. Figure 3c shows substantially increased IL- $1 \beta$ mRNA and protein expression in SIRT5 KO cells, a major downstream effector of the $\mathrm{PI} 3 \mathrm{~K} / \mathrm{AKT} / \mathrm{NF}-\mathrm{kB}$ signaling pathway. One of the upstream regulators of this pathway, PI3K, is limited by PTEN. PTEN is considered a tumor suppressor, and the PC-3 cell line possesses a PTEN mutation $(36,37)$. We also confirmed the lack of PTEN signal in each PC-3, SIRT5 KO, and PC-3M cell line using western blot (data not shown); nonetheless, we found increased levels of PI3K specifically in SIRT5 KO cells. Based on these interesting observations, we concentrated on our hypothesis that SIRT5 can control the PI3K/AKT/NF-kB signaling pathway.

$\mathrm{PI} 3 \mathrm{~K} / \mathrm{AKT} / \mathrm{NF}-\mathrm{kB}$ signaling pathway is involved in cell growth and proliferation in human cancer (38). In the cancer cells from human gallbladder and prostate, the phosphorylated AKT level was increased, causing fast cell growth, proliferation, and invasion (39). To confirm the DEP search data results, we performed western blots for PI3K on SIRT5 KO and PC-3 cells and verified that SIRT5 KO cells showed overexpression of PI3K but not TRAF2, another upstream regulator of the pathway (Figure 3b). Consequently, we devised a direct correlation between SIRT5 and PI3K, but not TRAF2, which was verified through the detection of SIRT5 by immunoprecipitation of PI3K (Figure 3d). The interaction between SIRT5 and PI3K modulated the downstream signaling system through major PI3K signaling components, including AKT phosphorylation and p50/p65, which coordinately regulates IL- $1 \beta$ expression and were all increased with the loss of SIRT5 (Figure 3f).

Interestingly, it was demonstrated that the SIRT5/PI3K axis mediated extremely high levels of IL-1 $\beta$ mRNA and protein in SIRT5 KO cells. Positive transcriptional regulators of this pathway, such as p50/65, were also induced in SIRT5 KO cells (40). IL-1 $\beta$ expression is positively correlated with bone metastasis in breast cancer, wherein IL-1 $\beta$ up-regulates VEGF and its corresponding receptor on endothelial cells to facilitate EC migration (41). In $\mathrm{PCa}$, the levels of testosterone and IL- $1 \beta$ showed a negative correlation, and thus androgen deprivation therapy does not mitigate the increased levels of IL-1 $\beta$ in patients with prostate cancer (42). Thus, it is suggested that the high 
level of IL- $1 \beta$ may be associated with PCa aggressiveness or recurrence. Although the androgen-independent PCa cell lines used in the experiments were not sensitive to testosterone levels, the high concentration of IL-1 $\beta$ in the cells affects PCa metastasis.

In conclusion, this study is the first to implicate SIRT5 as the only member of the SIRT family with decreased expression in metastatic prostate cancer. The expression of SIRT5 in this context is controlled by protein homeostasis processes rather than gene transcription. The loss of SIRT5 in aggressive $\mathrm{PCa}$ cell lines simultaneously increased $\mathrm{PI} 3 \mathrm{~K} / \mathrm{AKT} / \mathrm{NF}-\mathrm{kB}$ pathway activation and expression of IL$1 \beta \square$ The data are evidence that SIRT5 is involved in prostate cancer metastasis and may be used as a biomarker for metastasis or in the developing of metastatic inhibitors.

\section{Conflicts of Interest}

The Authors declare no competing interests.

\section{Authors' Contributions}

S.Y..C., and J.M.J., jointly carried out the experiment and prepared the article. A.Y.N., and O.K.K. helped to proteome data generation. I.H.B., E.J.B. and B.H.P provided the SIRT5 KO cell-line. Y.H., E.H.L. and T.G.K. provided the conceptual and technical quidance for prostate cancer. J.N.L. and S.L. jointly designed the study, and conceived the original idea supervied the project. All Authors read and approved the final article.

\section{Acknowledgements}

This work was supported by a National Research Foundation of Korea (NRF) grant funded by the Korean government (MSIP) (2019R1H1A1079839, 2020R1A2B5B03002344 and 2020M3A9B 6037812).

\section{References}

1 Frye RA: Phylogenetic classification of prokaryotic and eukaryotic Sir2-like proteins. Biochem Biophys Res Commun 273(2): 793-798, 2000. PMID: 10873683. DOI: 10.1006/bbrc. 2000.3000

2 Brachmann CB, Sherman JM, Devine SE, Cameron EE, Pillus L and Boeke JD: The SIR2 gene family, conserved from bacteria to humans, functions in silencing, cell cycle progression, and chromosome stability. Genes Dev 9(23): 2888-2902, 1995. PMID: 7498786. DOI: $10.1101 / \mathrm{gad} .9 .23 .2888$

3 Michishita E, Park JY, Burneskis JM, Barrett JC and Horikawa I: Evolutionarily conserved and nonconserved cellular localizations and functions of human SIRT proteins. Mol Biol Cell 16(10): 4623-4635, 2005. PMID: 16079181. DOI: 10.1091/mbc.e05-01-0033

4 Yeung F, Hoberg JE, Ramsey CS, Keller MD, Jones DR, Frye RA and Mayo MW: Modulation of NF-kappaB-dependent transcription and cell survival by the SIRT1 deacetylase. EMBO J 23(12): 23692380, 2004. PMID: 15152190. DOI: 10.1038/sj.emboj.7600244
5 Guarani V, Deflorian G, Franco CA, Krüger M, Phng LK, Bentley K, Toussaint L, Dequiedt F, Mostoslavsky R, Schmidt MHH, Zimmermann B, Brandes RP, Mione M, Westphal CH, Braun T, Zeiher AM, Gerhardt H, Dimmeler S and Potente M: Acetylation-dependent regulation of endothelial Notch signalling by the SIRT1 deacetylase. Nature 473(7346): 234-238, 2011. PMID: 21499261. DOI: 10.1038/nature09917

6 Malik S, Villanova L, Tanaka S, Aonuma M, Roy N, Berber E, Pollack JR, Michishita-Kioi E and Chua KF: SIRT7 inactivation reverses metastatic phenotypes in epithelial and mesenchymal tumors. Sci Rep 5: 9841, 2015. PMID: 25923013. DOI: 10.1038/ srep09841

7 Hirschey MD and Zhao Y: Metabolic regulation by lysine malonylation, succinylation, and glutarylation. Mol Cell Proteomics 14(9): 2308-2315, 2015. PMID: 25717114. DOI: 10.1074/mcp.R114.046664

8 Bringman-Rodenbarger LR, Guo AH, Lyssiotis CA and Lombard DB: Emerging roles for SIRT5 in metabolism and cancer. Antioxid Redox Signal 28(8): 677-690, 2018. PMID: 28707979. DOI: $10.1089 /$ ars 2017.7264

9 Wang C, Zhang C, Li X, Shen J, Xu Y, Shi H, Mu X, Pan J, Zhao T, Li M, Geng B, Xu C, Wen H and You Q: CPT1Amediated succinylation of S100A10 increases human gastric cancer invasion. J Cell Mol Med 23(1): 293-305, 2019. PMID: 30394687. DOI: $10.1111 / \mathrm{jcmm} .13920$

10 Wang YQ, Wang HL, Xu J, Tan J, Fu LN, Wang JL, Zou TH, Sun DF, Gao QY, Chen YX and Fang JY: Sirtuin5 contributes to colorectal carcinogenesis by enhancing glutaminolysis in a deglutarylation-dependent manner. Nat Commun 9(1): 545, 2018. PMID: 29416026. DOI: 10.1038/s41467-018-02951-4

11 Chang L, Xi L, Liu Y, Liu R, Wu Z and Jian Z: SIRT5 promotes cell proliferation and invasion in hepatocellular carcinoma by targeting E2F1. Mol Med Rep 17(1): 342-349, 2018. PMID: 29115436. DOI: $10.3892 / \mathrm{mmr} .2017 .7875$

12 Tang Z, Li L, Tang Y, Xie D, Wu K, Wei W and Xiao Q: CDK2 positively regulates aerobic glycolysis by suppressing SIRT5 in gastric cancer. Cancer Sci 109(8): 2590-2598, 2018. PMID: 29896817. DOI: $10.1111 /$ cas. 13691

13 Siegel RL, Miller KD and Jemal A: Cancer statistics, 2018. CA Cancer J Clin 68(1): 7-30, 2018. PMID: 29313949. DOI: $10.3322 /$ caac. 21442

14 Wan L, Pantel K and Kang Y: Tumor metastasis: moving new biological insights into the clinic. Nat Med 19(11): 1450-1464, 2013. PMID: 24202397. DOI: $10.1038 / \mathrm{nm} .3391$

15 Manca P, Pantano F, Iuliani M, Ribelli G, De Lisi D, Danesi R, Del Re M, Vincenzi B, Tonini G and Santini D: Determinants of bone specific metastasis in prostate cancer. Crit Rev Oncol Hematol 112: 59-66, 2017. PMID: 28325265. DOI: 10.1016/j.critrevonc.2017.02.013

16 Hong MK, Macintyre G, Wedge DC, Van Loo P, Patel K, Lunke S, Alexandrov LB, Sloggett C, Cmero M, Marass F, Tsui D, Mangiola S, Lonie A, Naeem H, Sapre N, Phal PM, Kurganovs N, Chin X, Kerger M, Warren AY, Neal D, Gnanapragasam V, Rosenfeld N, Pedersen JS, Ryan A, Haviv I, Costello AJ, Corcoran NM and Hovens CM: Tracking the origins and drivers of subclonal metastatic expansion in prostate cancer. Nat Commun 6: 6605, 2015. PMID: 25827447. DOI: 10.1038/ncomms7605

17 Cabello M, Ge H, Aracil C, Moschou D, Estrela P, Manuel Quero J, I Pascu S and R F Rocha P: Extracellular electrophysiology in the prostate cancer cell model PC-3. Sensors (Basel) 19(1): 139, 2019. PMID: 30609788. DOI: 10.3390/s19010139 
18 Pettaway CA, Pathak S, Greene G, Ramirez E, Wilson MR, Killion JJ and Fidler IJ: Selection of highly metastatic variants of different human prostatic carcinomas using orthotopic implantation in nude mice. Clin Cancer Res 2(9): 1627-1636, 1996. PMID: 9816342.

19 Perez-Riverol Y, Csordas A, Bai J, Bernal-Llinares M, Hewapathirana S, Kundu DJ, Inuganti A, Griss J, Mayer G, Eisenacher M, Pérez E, Uszkoreit J, Pfeuffer J, Sachsenberg T, Yilmaz S, Tiwary S, Cox J, Audain E, Walzer M, Jarnuczak AF, Ternent T, Brazma A and Vizcaíno JA: The PRIDE database and related tools and resources in 2019: improving support for quantification data. Nucleic Acids Res 47(D1): D442-D450, 2019. PMID: 30395289. DOI: 10.1093/nar/gky1106

20 Cox J and Mann M: MaxQuant enables high peptide identification rates, individualized p.p.b.-range mass accuracies and proteomewide protein quantification. Nat Biotechnol 26(12): 1367-1372, 2008. PMID: 19029910. DOI: 10.1038/nbt.1511

21 Tyanova S, Temu T, Sinitcyn P, Carlson A, Hein MY, Geiger T, Mann M and Cox J: The Perseus computational platform for comprehensive analysis of (prote)omics data. Nat Methods 13(9): 731-740, 2016. PMID: 27348712. DOI: 10.1038/nmeth.3901

22 Huang da W, Sherman BT and Lempicki RA: Systematic and integrative analysis of large gene lists using DAVID bioinformatics resources. Nat Protoc 4(1): 44-57, 2009. PMID: 19131956. DOI: $10.1038 /$ nprot.2008.211

23 Szklarczyk D, Franceschini A, Wyder S, Forslund K, Heller D, Huerta-Cepas J, Simonovic M, Roth A, Santos A, Tsafou KP, Kuhn M, Bork P, Jensen LJ and von Mering C: STRING v10: protein-protein interaction networks, integrated over the tree of life. Nucleic Acids Res 43(Database issue): D447-D452, 2015. PMID: 25352553. DOI: 10.1093/nar/gku1003

24 Ohnuki Y, Marnell MM, Babcock MS, Lechner JF and Kaighn ME: Chromosomal analysis of human prostatic adenocarcinoma cell lines. Cancer Res 40(3): 524-534, 1980. PMID: 7471073.

25 Cheung AK, Ko JM, Lung HL, Chan KW, Stanbridge EJ, Zabarovsky E, Tokino T, Kashima L, Suzuki T, Kwong DL, Chua D, Tsao SW and Lung ML: Cysteine-rich intestinal protein 2 (CRIP2) acts as a repressor of NF-kappaB-mediated proangiogenic cytokine transcription to suppress tumorigenesis and angiogenesis. Proc Natl Acad Sci USA 108(20): 8390-8395, 2011. PMID: 21540330. DOI: 10.1073/pnas.1101747108

26 Huang Z, Zhuo Y, Shen Z, Wang Y, Wang L, Li H, Chen J and Chen W: The role of NEFL in cell growth and invasion in head and neck squamous cell carcinoma cell lines. J Oral Pathol Med 43(3): 191-198, 2014. PMID: 23992471. DOI: 10.1111/jop.12109

27 Lekstrom-Himes J and Xanthopoulos KG: Biological role of the CCAAT/enhancer-binding protein family of transcription factors. J Biol Chem 273(44): 28545-28548, 1998. PMID: 9786841. DOI: $10.1074 /$ jbc. 273.44 .28545

28 Nutter F, Holen I, Brown HK, Cross SS, Evans CA, Walker M, Coleman RE, Westbrook JA, Selby PJ, Brown JE and Ottewell PD: Different molecular profiles are associated with breast cancer cell homing compared with colonisation of bone: evidence using a novel bone-seeking cell line. Endocr Relat Cancer 21(2): 327-341, 2014. PMID: 24413608. DOI: 10.1530/ERC-13-0158

29 Cogswell JP, Godlevski MM, Wisely GB, Clay WC, Leesnitzer LM, Ways JP and Gray JG: NF-kappa B regulates IL-1 beta transcription through a consensus NF-kappa B binding site and a nonconsensus CRE-like site. J Immunol 153(2): 712-723, 1994. PMID: 8021507.
30 Bello D, Webber MM, Kleinman HK, Wartinger DD and Rhim JS: Androgen responsive adult human prostatic epithelial cell lines immortalized by human papillomavirus 18. Carcinogenesis 18(6): 1215-1223, 1997. PMID: 9214605. DOI: 10.1093/carcin/ 18.6.1215

31 Rasti N, Wahlgren M and Chen Q: Molecular aspects of malaria pathogenesis. FEMS Immunol Med Microbiol 41(1): 9-26, 2004. PMID: 15094163. DOI: 10.1016/j.femsim.2004.01.010

32 Chalkiadaki A and Guarente L: The multifaceted functions of sirtuins in cancer. Nat Rev Cancer 15(10): 608-624, 2015. PMID: 26383140. DOI: 10.1038/nrc3985

$33 \mathrm{Lu} \mathrm{W}$, Zuo Y, Feng Y and Zhang M: SIRT5 facilitates cancer cell growth and drug resistance in non-small cell lung cancer. Tumour Biol 35(11): 10699-10705, 2014. PMID: 25070488. DOI: $10.1007 / \mathrm{s} 13277-014-2372-4$

34 Igci M, Kalender ME, Borazan E, Bozgeyik I, Bayraktar R, Bozgeyik E, Camci C and Arslan A: High-throughput screening of Sirtuin family of genes in breast cancer. Gene 586(1): 123128, 2016. PMID: 27080717. DOI: 10.1016/j.gene.2016.04.023

35 Lai CC, Lin PM, Lin SF, Hsu CH, Lin HC, Hu ML, Hsu CM and Yang MY: Altered expression of SIRT gene family in head and neck squamous cell carcinoma. Tumour Biol 34(3): 1847-1854, 2013. PMID: 23475622. DOI: 10.1007/s13277-013-0726-y

36 Vlietstra RJ, van Alewijk DC, Hermans KG, van Steenbrugge GJ and Trapman J: Frequent inactivation of PTEN in prostate cancer cell lines and xenografts. Cancer Res 58(13): 2720-2723, 1998. PMID: 9661880.

37 Russell PJ and Kingsley EA: Human prostate cancer cell lines. Methods Mol Med 81: 21-39, 2003. PMID: 12725112. DOI: 10.1385/1-59259-372-0:21

38 Lim HJ, Crowe P and Yang JL: Current clinical regulation of $\mathrm{PI} 3 \mathrm{~K} / \mathrm{PTEN} / \mathrm{Akt} / \mathrm{mTOR}$ signalling in treatment of human cancer. J Cancer Res Clin Oncol 141(4): 671-689, 2015. PMID: 25146530. DOI: 10.1007/s00432-014-1803-3

39 Li X, Teng S, Zhang Y, Zhang W, Zhang X, Xu K, Yao H, Yao J, Wang $\mathrm{H}$, Liang $\mathrm{X}$ and $\mathrm{Hu} \mathrm{Z}$ : TROP2 promotes proliferation, migration and metastasis of gallbladder cancer cells by regulating PI3K/AKT pathway and inducing EMT. Oncotarget 8(29): 4705247063, 2017. PMID: 28423362. DOI: 10.18632/oncotarget.16789

40 Qi JC, Xue WY, Zhang YP, Qu CB, Lu BS, Yin YW, Liu KL, Wang DB, Li W and Zhao ZM: Cholinergic $\alpha 5$ nicotinic receptor is involved in the proliferation and invasion of human prostate cancer cells. Oncol Rep 43(1): 159-168, 2020. PMID: 31789411. DOI: 10.3892/or.2019.7411

41 Yamamoto Y and Gaynor RB: Role of the NF-kappaB pathway in the pathogenesis of human disease states. Curr Mol Med 1(3): 287296, 2001. PMID: 11899077. DOI: 10.2174/1566524013363816

42 Tulotta C and Ottewell P: The role of IL-1B in breast cancer bone metastasis. Endocr Relat Cancer 25(7): R421-R434, 2018. PMID: 29760166. DOI: 10.1530/ERC-17-0309

43 Maggio M, Basaria S, Ceda GP, Ble A, Ling SM, Bandinelli S, Valenti $G$ and Ferrucci L: The relationship between testosterone and molecular markers of inflammation in older men. J Endocrinol Invest 28(11 Suppl Proceedings): 116-119, 2005. PMID: 16760639.

Received September 14, 2021

Revised October 15, 2021

Accepted November 17, 2021 